\title{
La construcción del catálogo de paisaje urbano del Parque \\ Sarmiento de la ciudad de Córdoba
}

\section{The construction of townscape catalog of Sarmiento Park in Córdoba city}

\begin{abstract}
Lucas Períes*
Silvina de Lourdes Barraud*

Recibido: mayo 18 de 2020.

Aceptado: septiembre 22 de 2020.

Resumen

Este artículo expone los resultados de la investigación realizada en el emblemático Parque Sarmiento de la ciudad de Córdoba, Argentina. Para su concreción, se aplica un procedimiento inédito, definido por el equipo de investigación en una serie de trabajos concatenados en los que participan profesionales de múltiples disciplinas. El trabajo concluye con la generación de un catálogo de paisaje del Parque Sarmiento, con la determinación del carácter del paisaje y sus valores. La investigación se lleva adelante a partir de una metodología compuesta por una serie de fases organizadas sistemáticamente y articuladas entre sí: interpretación, identificación, caracterización, valoración y evaluación. El proceso completo permite establecer las consideraciones de calidad paisajística, así como las respectivas acciones que demandan para el desarrollo sostenible del paisaje.
\end{abstract}

Palabras clave: espacio urbano, valoración, caracterización, catalogación, morfología urbana.

\begin{abstract}
This article presents the results of the research carried out in the emblematic Sarmiento Park in Cordoba city, Argentina. For its concretion, an unpublished procedure is applied, defined by the research team in a series of concatenated works in which professionals from multiple disciplines participate. The work concludes with the generation of a landscape catalog of Sarmiento Park, with the determination of the landscape's character and its values. The research is carried out based on a methodology composed of a series of phases organized systematically and articulated among themselves: interpretation, identification, characterization, assessment, and evaluation. The complete process allows establishing the landscape quality considerations as well as the respective actions that demand the sustainable development of the landscape.
\end{abstract}

Keywords: Urban space, assessment, characterization, cataloging, urban morphology.

*Universidad Católica de Córdoba, Unidad Asociada al CONICET, Córdoba, Argentina. Correos electrónicos: perieslucas@gmail.com, sbarraud2001@gmail.com 


\section{Introducción}

La construcción del catálogo de paisaje del Parque Sarmiento es resultado de la investigación aplicada que se enmarca en la línea de investigación de Catálogos de Paisaje Urbano (CPU), la cual trabaja en la definición metodológica de los mismos y es desarrollada por más de diez años. Estos catálogos constituyen un instrumento innovador, generado para orientar los procesos de planificación urbana con un enfoque paisajístico, y en referencia al desarrollo sostenible, tal como lo plantean Busquets y Cortina: la sostenibilidad paisajística es la "capacidad de un cierto paisaje de sostener las actividades de la sociedad sin comprometer el mantenimiento de sus características y valores esenciales" (Busquets y Cortina, 2009: 702).

La finalidad de los CPU es determinar el tipo de valor que posee un paisaje y establecer las consideraciones de calidad paisajística que permitan delinear acciones para su manutención y desarrollo - en términos de calidad de vida urbana-, todo ello orientado a contribuir con la mejora de la gestión, el análisis y la utilización de los recursos naturales y culturales, para la formulación de planes, proyectos y políticas urbanas, desde el bagaje técnico-conceptual del paisaje y con abordaje interdisciplinar.

El diseño y la ejecución de un CPU constituyen un mecanismo inédito frente a otros antecedentes que abordan la planificación territorial a escala regional por medio de estrategias análogas, principalmente las desarrolladas en Europa. Nuestra investigación apunta a la generación de un instrumento de acción propio que posibilite aplicaciones al contexto y a la realidad latinoamericana, y con escala y delimitación en el ámbito de la ciudad.

Este artículo deriva de la investigación citada y realizada en el ámbito del Instituto del Paisaje de la Universidad Católica de Córdoba-Unidad, asociada al Consejo Nacional de Investigaciones Científicas y Técnicas, dependiente del Ministerio de Ciencia, Tecnología e Innovación Productiva de Argentina (CONICET), con subsidios de la propia institución y evaluación de jurado externo. La autoría corresponde a profesionales de la Arquitectura - con formación en carreras de posgrado complementarias- y es coincidente con las personas responsables del proyecto. Este equipo cuenta con la colaboración de múltiples especialistas provenientes de los campos de la Biología, Geología, Ciencias Agronómicas, Diseño Industrial y Artes plásticas, quienes realizan sus aportes específicos e integrados en distintas instancias de la labor científica. Además, colaboran estudiantes de la carrera de Arquitectura UCC.

La investigación plantea como objetivo general la definición metodológica de la construcción de los CPU para su aplicación en distintas estructuras físico-espaciales urbanas y contextos geográficos. En lo específico se propone ejecutar la estrategia procedimental de abordaje del caso de aplicación (Parque Sarmiento) contribuyendo al conocimiento de su estado y valor paisajístico. Ambos propósitos se han alcanzado y el sentido de este artículo es exponer la síntesis de los procedimientos desarrollados y del producto resultante. 
Para tal fin, el trabajo se fundamenta en la propia investigación aplicada -desde la producción original- y se sustenta en antecedentes externos - algunos de los cuales se referencian en el desarrollo del escrito-. La posibilidad de hacer pública esta experiencia posibilitará que pueda ser replicada o reelaborada por profesionales con interés en el tema, al mismo tiempo que su empleo pretende aportar como material didáctico en el campo de la enseñanza.

A continuación, se reseña la condición del Parque Sarmiento y posteriormente se desarrolla la metodología de construcción del CPU, haciendo explícitas las técnicas y herramientas empleadas, en simultáneo con los resultados obtenidos en el caso de aplicación.

\section{El Parque Sarmiento}

El Parque Sarmiento constituye un referente nacional en lo que a parques urbanos respecta, cuyos orígenes datan de finales del siglo XIX. Está ubicado en una situación próxima al centro geográfico de la ciudad de Córdoba; sus inmediaciones Norte y Oeste se caracterizan, actualmente, por una importante densidad demográfica.

Alberga importantes museos, centros culturales, educativos y gastronómicos, teatros, espacios recreativos y deportivos, plazas, plazoletas, jardines y paseos con monumentos y obras artísticas de diferentes épocas y autores; todo ello se encuentra junto a un gran jardín zoológico e importante volumen de vegetación nativa y exótica. Por otra parte, las condiciones geográficas de su emplazamiento constituyen un patrimonio natural de gran significancia. Al respecto, el parque presenta una topografía de terrazas con un plano alto y algunas ondulaciones puntuales, el cual está interrumpido por un quiebre ocasionado por barrancas con fuerte desnivel, en las que predominan relictos de vegetación y fauna nativa. Las barrancas son elementos testimoniales y únicos del paisaje originario donde se sitúa la ciudad.

El Parque Sarmiento cuenta, fundamentalmente, con gran valor patrimonial, además de los motivos expuestos, por ser el primer parque público proyectado (1889) y construido en el país por el arquitecto paisajista francés Carlos Thays, profesional sobre el que Page expone:

En la ciudad-luz trabajó con el afamado paisajista Edouard André, profesor de la escuela de Versalles, atendiendo diversos proyectos en toda Europa, en los tiempos que gobernaba Napoleón III en aquel agitado “Segundo Imperio". Allí conoció al empresario argentino Miguel Crisol quien le comunicó que por indicación del ingeniero Alphand, director de los trabajos de transformación de París y uno de sus principales maestros, se dirigiera a él para que proyectara un parque de Córdoba (Page, 1996: 13). 
Las cuestiones reseñadas, sumadas al elevado grado de deterioro general y la presencia de sectores en extremo abandono, son los motivos que en 2017 propiciaron la declaratoria de Monumento Histórico Nacional -con carácter de urgencia-, más de cien años después de su concepción. La investigación que se realiza, aplicada en este espacio urbano, se encamina a la producción de un aporte específico y detallado sobre el conocimiento de su realidad actual -los componentes y su dinámica, el carácter del paisaje, el estado de conservación y los valores paisajísticos-, y su prospectiva de desarrollo sostenible como posibilidades para la práctica, en su transferencia a gobiernos locales. Al mismo tiempo, permite contribuir a la teoría de la Planificación del Paisaje territorialurbano.

\section{Metodología y resultados del Catálogo de Paisaje Urbano}

El proceso metodológico para la construcción del CPU se estructura en cinco fases consecutivas: interpretación, identificación, caracterización, valoración y evaluación. Este esquema es resultado de la investigación continua en el tema, y toma como base referencial a los distintos proyectos que surgen a partir del Convenio Europeo del Paisaje (Consejo de Europa, 2000), principalmente la estrategia para Catálogos del paisaje desarrollada por el Observatorio del Paisaje de Cataluña (Nogué, Sala, Grau, 2016), la Guía de evaluación del carácter del paisaje para Inglaterra y Escocia (Swanwick, 2002) de Scottish Natural Heritage y el proyecto Pays.doc de cooperación transnacional liderado por la Junta de Andalucía e integrado por organismos de Italia, Francia, Grecia y España (Busquets, 2007).

\section{La fase de interpretación}

La interpretación apunta al entendimiento del territorio urbano y de la estructura físicoespacial de la zona de estudio. Las actividades consisten en el reconocimiento y el análisis de documentación (gráfica, fotográfica y escrita) y material bibliográfico, así como a la consulta de historiadores y especialistas en el caso para producir una descripción de la secuencia histórica de la construcción del paisaje. Para ello, se consideran hechos y situaciones significativas que determinan su condición actual. Esta fase permite aproximarse al entendimiento de la estructura físico-espacial de la zona de estudio.

La interpretación territorial se completa con el estudio morfológico del espacio urbano. La zona de estudio se interpreta mediante una reducción sintética de su complejidad y en un análisis de restricción formal - que se escinde de otros aspectos o indicadores-; para ello, se analizan los siguientes cuatro elementos: trazados, manzanas, edificios y arbolado. En este sentido, el resultado se expone en la figura 2 y en las siguientes argumentaciones. 
Figura 1. Mapas de localización de la zona de estudio
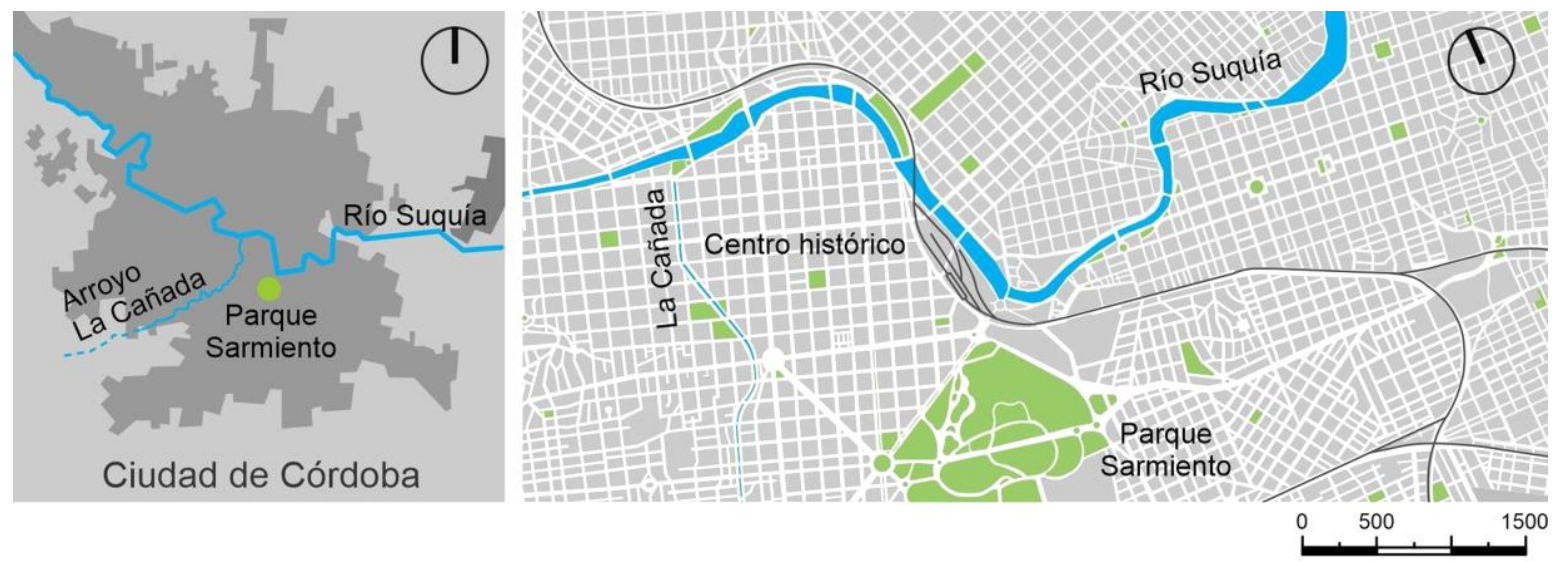

Fuente: elaboración propia.

Figura 2. Esquemas interpretativos de la morfología del espacio urbano de la zona de estudio

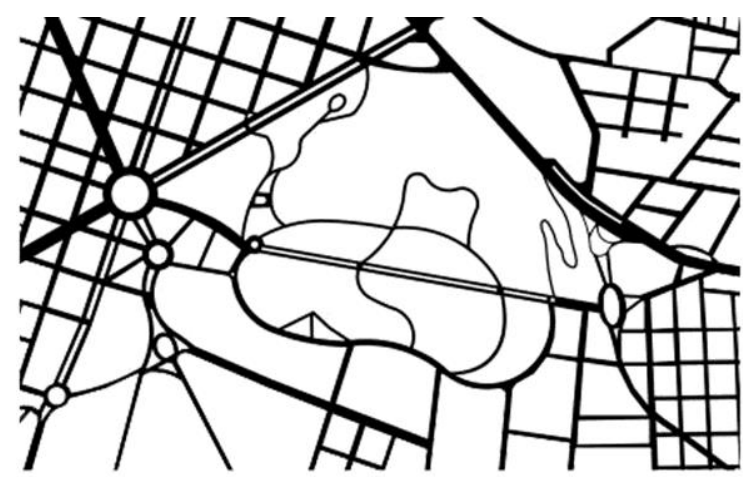

SISTEMADE TRAZADOS

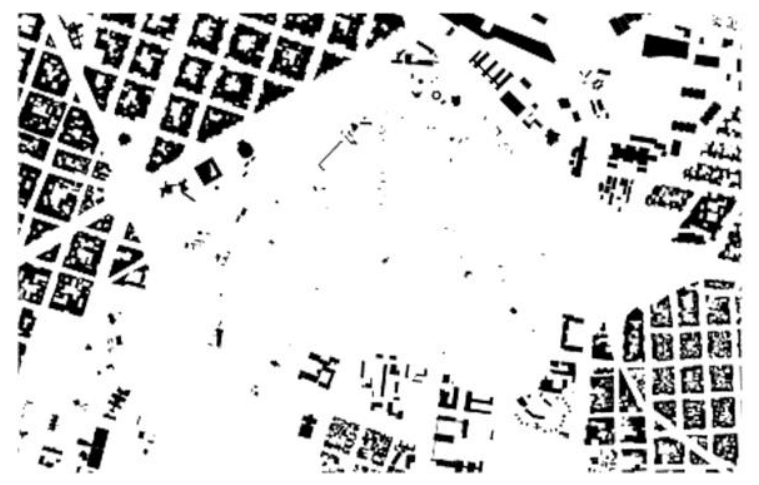

SISTEMIA EDIFICATORIO

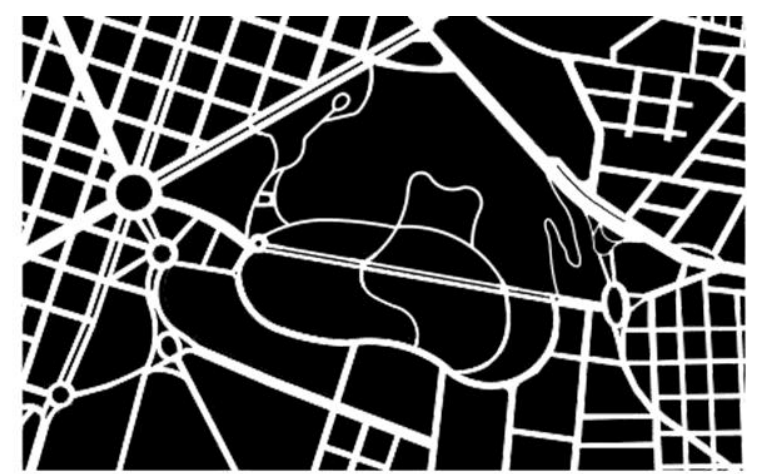

SISTEMIA DE AMIANZANAMIENTO

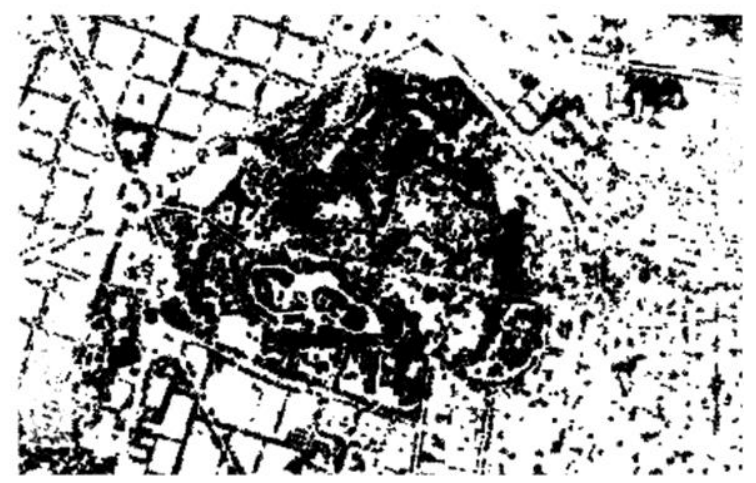

SISTEMA DE ARBOLADO 
- Las infraestructuras viarias y de ordenación se interpretan por medio de tres tipos de trazados, los cuales, en determinadas ocasiones, se presentan articulados: ortogonal (trazado con predominio de infraestructuras viarias rectas que se cruzan o interceptan en ángulo recto), curvilíneo (trazado con predominio de infraestructuras viarias curvas que se cruzan o interceptan) y radial (trazado con predominio de infraestructuras viarias rectas y curvas que se disponen de manera radial con convergencia a puntos); todo ello es consecuencia de la extensión de la urbanización cuadricular del sistema colonial, las diagonales del siglo XIX y el diseño del propio Parque Sarmiento.

- El sistema de amanzanamiento difiere según los tipos de trazados en cuanto a las distintas figuras de manzana presentes: cuadrangulares, rectangulares, trapezoidales, triangulares, circulares, polígonos irregulares, figuras curvas irregulares y figuras mixtas (con lados rectos y curvos). Al mismo tiempo, se reconoce gran variación de tamaño, principalmente en el sector de urbanización curvilíneo correspondiente con el Parque Sarmiento y Ciudad Universitaria.

- El sistema edificatorio corresponde directamente al tipo de amanzanamiento. Las manzanas ortogonales se consolidan con altos porcentajes de ocupación perimetral dejando vacíos interiores; las figuras responden a la lógica geométrica ortogonal. En el caso de las grandes manzanas se presentan edificaciones aisladas con figuras y tamaños diversos.

- El arbolado se interpreta en directa relación con los trazados y las manzanas. En los trazados de tipo ortogonal y radial, los árboles se disponen de manera alineada en correspondencia con la infraestructura viaria, en el perímetro de las manzanas y con mínima presencia en sus interiores. En el trazado curvilíneo, la alineación a la infraestructura viaria disminuye y aparecen agrupaciones arbóreas o grandes superficies de manchas boscosas irregulares en el interior de las manzanas.

Finalmente, como cierre de la fase de interpretación, se construye un plano de subdivisión y distribución de la espacialidad física de la zona de estudio -con sus respectivas denominaciones-, representativo de la situación actual y que se constituye como la herramienta de base referencial para la continuidad del trabajo en las fases consecutivas. 


\section{La fase de identificación}

La identificación se inicia con la determinación de cuencas visuales, ${ }^{1}$ a partir de la localización de puntos de observación ${ }^{2}$ para la captura de fotografías en formato de barridos panorámicos. ${ }^{3}$ Esto permite producir una mirada general y abarcadora de la zona de estudio y con las fotografías se pueden analizar los planos de visibilidad -esquemas interpretativos de la visión representados como capas que dividen la visual del paisaje según distintas profundidades-, el color ambiental -medición colorimétrica promedio de la imagen paisajística- y la fisonomía de cuencas visuales.

En el Parque Sarmiento se determinan 18 puntos de observación para la captura de barridos panorámicos (fotografías de $360^{\circ}$ ) -en los periodos de otoño y primavera-, como se expone en la figura 3. Su localización se establece teniendo en cuenta los siguientes criterios, definidos en correspondencia con la extensión del caso de estudio y el tipo de espacio urbano: a) lugares accesibles y preferentemente significativos; b) espacios abiertos y de mucha afluencia de personas (preferentemente dispuestos sobre infraestructura viaria); c) proximidad inferior a 300 metros entre PO, superponiendo los radios de miradas próximas (200 metros) para garantizar que todos los componentes del parque se identifiquen como unidad (salvo los de las categorías Flora y Mobiliario); d) altitud constante respecto del plano de suelo; e) incorporación de espacios del entorno del propio parque, como parte de la zona de estudio, para evitar un enfoque aislado del caso.

Luego se produce el reconocimiento de la composición del paisaje, por medio de un sistema de categorización de componentes paisajísticos que incluye aquellos tangibles e intangibles, como: flora, fauna, geomorfología, edificaciones, infraestructura, mobiliario, vehículos, actividades, eventos y materias. Para la identificación de los componentes, se plantea la distinción entre componentes naturales y antrópicos, estáticos y dinámicos, así como entre componentes lejanos. ${ }^{4}$ Con base en estos cinco grandes grupos de componentes, se propone categorías, subcategorías y tipos para su identificación pormenorizada. ${ }^{5}$

\footnotetext{
${ }^{1}$ La cuenca visual es la porción del territorio visible desde un punto de observación, en una situación temporal específica.

${ }^{2}$ El punto de observación es el lugar desde el cual se percibe principalmente al paisaje por sus condiciones de accesibilidad y visibilidad.

${ }^{3}$ El barrido panorámico es un tipo específico de fotografía gran angular, con formato de rectángulo alargado, correspondiente a un campo de visión ultra amplio $\left(360^{\circ}\right)$.

${ }^{4}$ Se consideran componentes lejanos aquellos de condición natural, cultural o mixta que se perciben a la distancia (lejanía) por fuera del área de influencias de una cuenca visual.

${ }^{5}$ Los criterios que sustentan el esquema de categorización de componentes paisajísticos son resultado de un extenso estudio de perfeccionamiento que comprende varios proyectos de investigación, donde participan profesionales diversos, para asegurar la inclusión y la distinción del mayor número posible de componentes, desde una perspectiva interdisciplinar. En el artículo "La identificación de componentes paisajísticos para catálogos del paisaje urbano" (Períes, Ojeda y Kesman, 2016), se muestra detalladamente.
} 
Figura 3. Localización de puntos de observación sobre imagen satelital

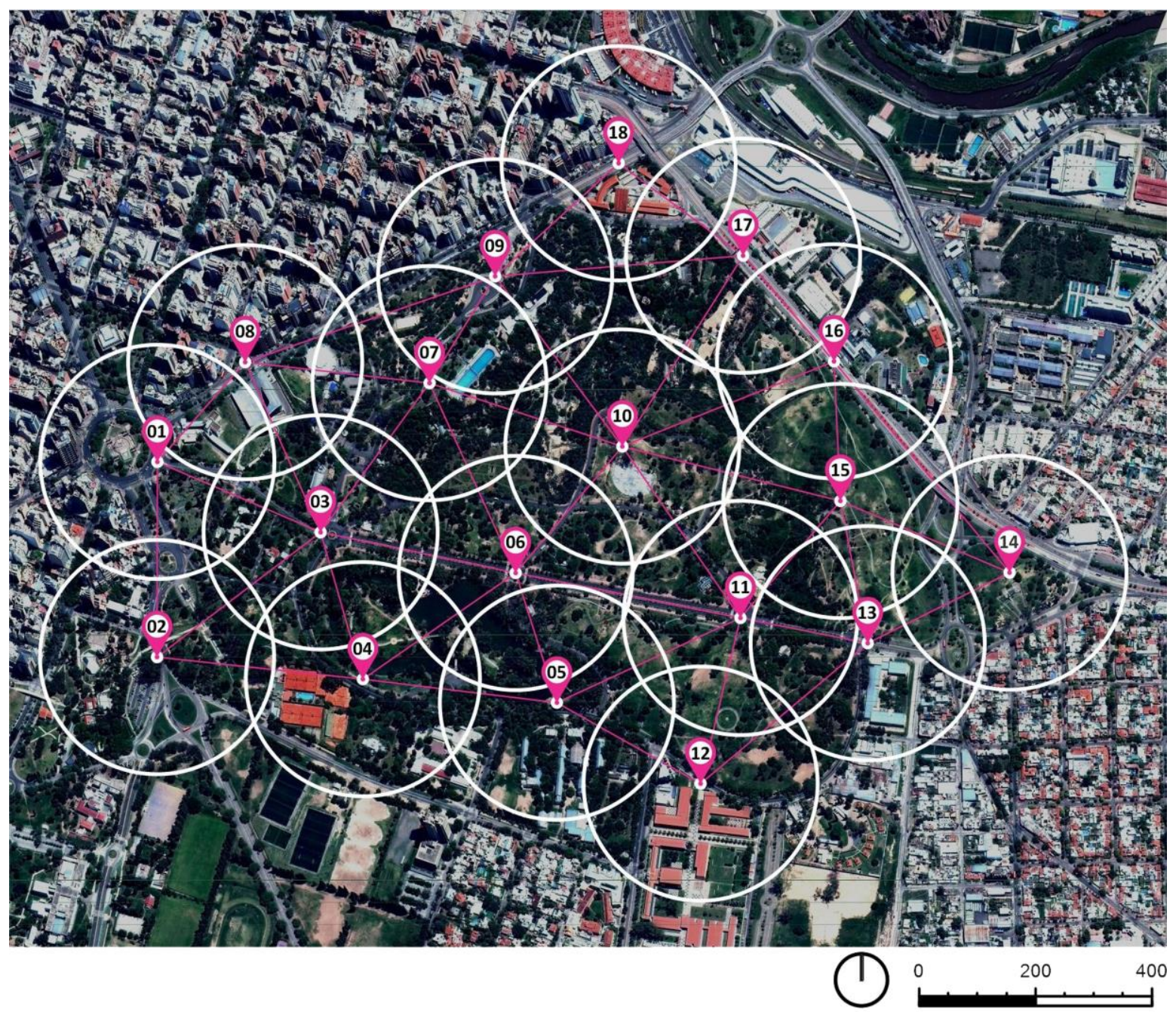

Fuente: elaboración propia sobre imagen satelital de la Dirección de Catastro de la Municipalidad de Córdoba.

Para la identificación de los componentes paisajísticos (CP) del Parque Sarmiento se establecen los siguientes criterios: a) los CP que se encuentran en un radio de 200 metros de cada punto de observación se identifican como unidad (excepto los correspondientes a las categorías de Flora y Mobiliario); b) se identifican solamente los CP que se localicen fuera del radio de 200 metros si se visualizan en los barridos panorámicos (como parte constitutiva de la imagen); c) si un CP es visualizado en el barrido panorámico y se localiza a más de 600 metros del punto de observación, se considera componente lejano y se emplea esa categoría.

Las fotografías panorámicas permiten hacer una lectura analítica de los elementos que constituyen cada cuenca visual del paisaje; se señalan los componentes en correspondencia a las categorías citadas, como se ejemplifica en la figura 4 . A cada categoría y subcategoría le corresponde un icono de identificación con el propio texto de referencia, 
donde se describe el tipo al que pertenece. La tarea se complementa con recorridos de campo y registros fotográficos de detalles, horarios, días y estaciones diferentes a la situación instantánea registrada en la panorámica. También se incluyen actividades de gabinete utilizando la tecnología "Streetview" de Google Maps. Con este procedimiento se identifican 1,832 componentes en el Parque Sarmiento, a un promedio de 98.8 componentes por cuenca visual.

Figura 4. Ejemplo de identificación de componentes paisajísticos sobre fotografía panorámica

Componentes estáticos del paisaje

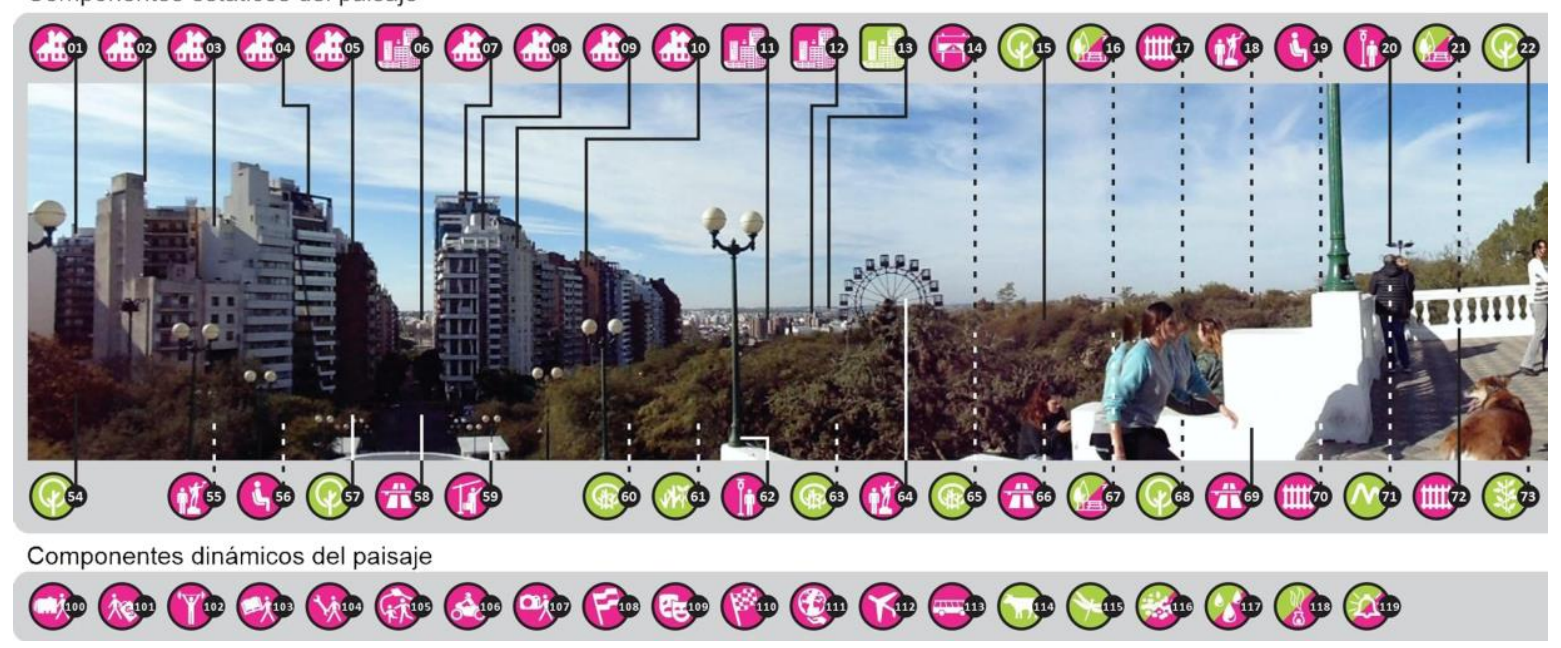

Fuente: elaboración propia.

La fase de identificación da como resultado un sistema de fichas correspondientes con las 18 cuencas visuales, en el que se vincula la información escrita, gráfica y fotográfica generada en las actividades expuestas. Las fichas se componen por los siguientes elementos: numeración de cuencas visuales, denominación de los puntos de observación y coordenadas geográficas de localización; mapa de localización del punto de observación, fotografía de aproximación al punto de observación, mapa de la cuenca visual sobre imagen satelital; fotografía de barrido panorámico; iconos y numeración de identificación de los componentes paisajísticos; análisis colorimétrico de las fotografías panorámicas en otoño y primavera; esquema de planos de visibilidad; listado de identificación de CP y fotografías de CP destacados. La figura 5 ejemplifica el formato de las fichas.

El material procesado constituye la plataforma inicial para la construcción del catálogo; produce la documentación del patrimonio natural y cultural, y tangible e intangible, que integra la zona de estudio. Si bien las fichas de identificación habilitan la ejecución de las fases sucesivas, en sí mismas constituyen un documento de relevamiento detallado que puede ser utilizado en otros marcos metodológicos de estudio e investigaciones o prácticas profesionales. 
Figura 5. Ejemplo de fichas de identificación del paisaje

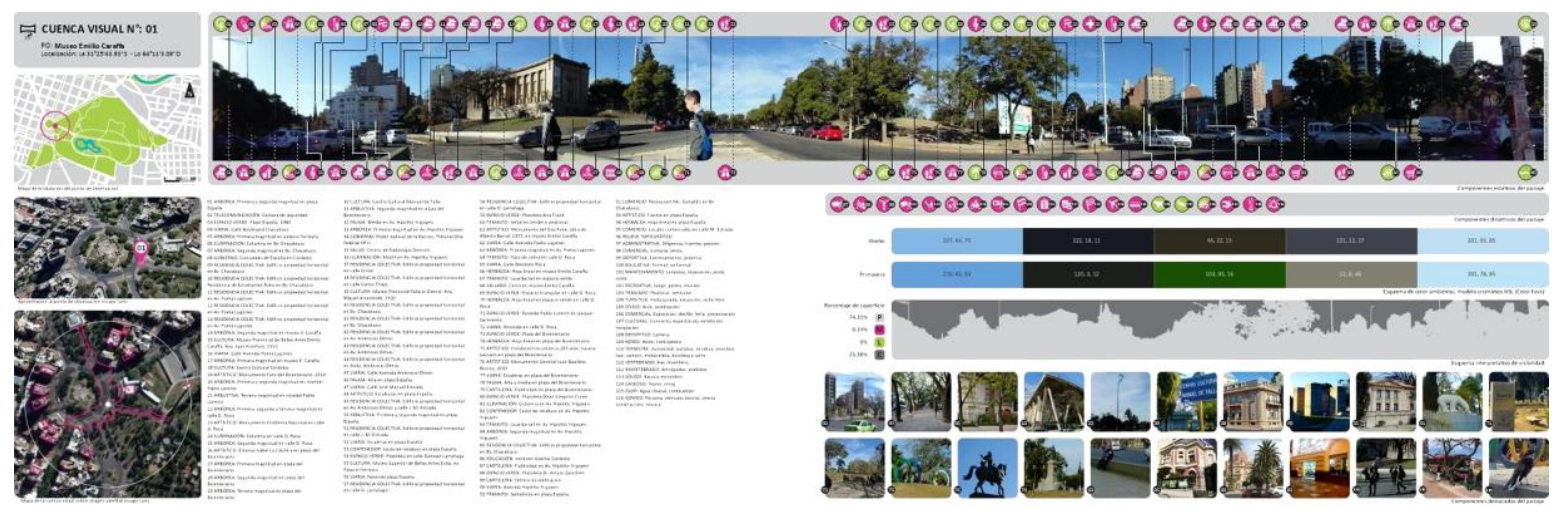

Fuente: elaboración propia.

\section{La fase de caracterización}

La caracterización realiza el reconocimiento de las áreas homogéneas de la zona de estudio en cuanto a la determinación del carácter del paisaje; este último deriva de un análisis pormenorizado de cada uno de los componentes paisajísticos que lo integran y en su interrelación y temporalidad. Al respecto, Porcal-Gonzalo establece que "el paisaje y su carácter van cambiando con el tiempo, aunque a menudo conserven unos rasgos heredados" (Porcal-Gonzalo, 2019: 227); la conjunción de la impronta histórica con la circunstancia actual conlleva a la determinación del carácter del paisaje.

La contrastación de la información correspondiente a cada cuenca visual permite establecer niveles de homogeneidad que determinan la subdivisión de la zona de estudio en áreas con caracteres diferenciados, tal como lo plantean Fernández y Plaza: "con ello se busca la cualidad o cualidades que individualizan y singularizan una parte del paisaje respecto de otra (el carácter)" (Fernández y Plaza, 2019: 268).

A partir del trabajo de identificación, se produce un proceso de profundización en la descripción de rasgos distintivos que caracterizan a los componentes registrados en las fichas de identificación. Para esta tarea, se dispone de una herramienta informática constituida por una base de datos con un sistema de tablas que procesa la caracterización. Las tablas están organizadas a partir de variables e indicadores según la información específica correspondiente a cada categoría de componentes paisajísticos. Operativamente, se analiza de modo pormenorizado cada uno de los componentes que integran las 18 fichas. 
En primer lugar, y según la categoría respectiva, se determina la subcategoría y el tipo que especifica al componente. Luego se desagregan las variables - sobre aspectos morfológicos, funcionales y de conservación, en términos cuantitativos y cualitativos- por medio de una serie de indicadores para cada una de ellas, tendientes a profundizar su descripción. El mismo procedimiento se realiza para los estudios de color ambiental,6 así como para el cálculo de cada cuenca visual y los planos de visibilidad, que se incluyen en cada ficha. En esta instancia se analizan los 1,832 componentes, los cuales son procesados a través de un promedio de quince variables, con un promedio también de cinco indicadores cada una -la mediación informática permite procesar el gran volumen de datos-.

Mediante el software se registra de manera numérica la cantidad de variables e indicadores seleccionados para cada categoría de componentes de una cuenca visual. El procesamiento siguiente consiste en la comparación por pares de cuencas visuales contiguas para observar los datos de categorías iguales. Dicha comparación establece la cantidad de tipos, indicadores y variables coincidentes o diferentes y sobre el total de componentes que integran la cuenca visual se calcula un porcentaje de cambio. De este modo, la comparación de datos entre todas las cuencas visuales señala la recurrencia o variabilidad de aspectos que caracterizan a las categorías de componentes para determinar las áreas homogéneas de la zona de estudio, desde aquellas cuencas que comparten características similares.

El procesamiento de datos que sigue este sistema informático permite circunscribir el carácter de cada cuenca visual, que está asociado, a través de concurrencias o divergencias, en la definición, la delimitación y la caracterización de las áreas homogéneas dentro de una zona de estudio. El resultado son cinco aéreas, a partir de asociar los espacios de influencias de las cuencas visuales con carácter homogéneo, como se expone en la figura 6. Un área homogénea se compone de combinaciones específicas de diversos componentes del paisaje que comparten características similares y confieren una marcada idiosincrasia por distinción con el resto del espacio urbano estudiado.

El carácter de cada área es desarrollado en particular por medio de documentos escritos, los cuales incluyen información cuantitativa, cualitativa y fotográfica; destacan aquellas categorías de componentes paisajísticos más relevantes - por su protagonismo o predominio-que determinan el carácter del paisaje de las áreas.

\footnotetext{
${ }^{6}$ Se puede acceder a los estudios de color en los CPU de manera detallada en el artículo "El color como componente paisajístico en los catálogos de paisaje urbano" (Períes, Kesman y Barraud, 2019).
} 


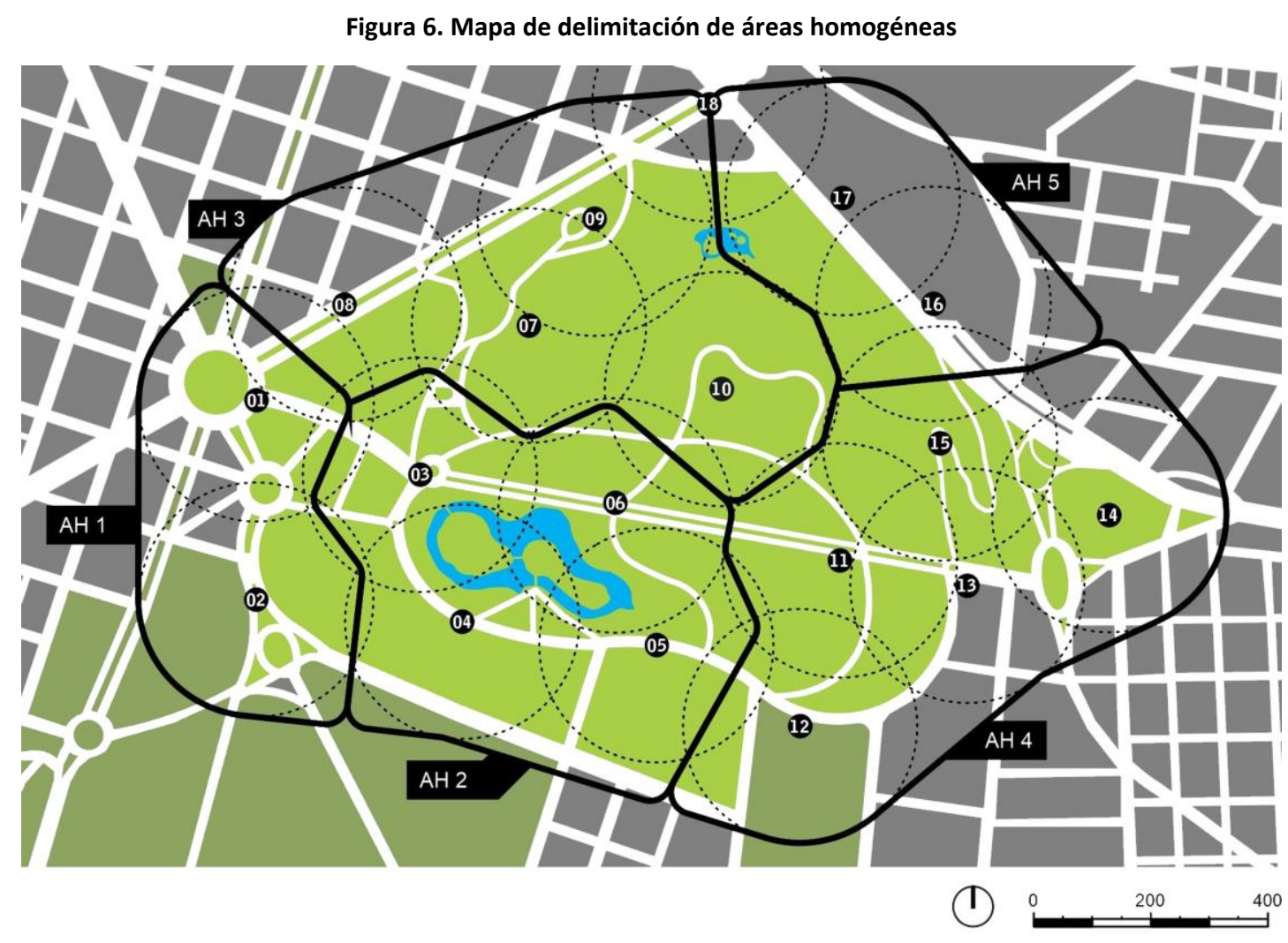

Fuente: elaboración propia.

\section{La fase de valoración}

La valoración se orienta al reconocimiento y a la asignación de valores a las áreas homogéneas detectadas en la caracterización. "Valorar el paisaje implica identificar, reconocer, caracterizar e interpretar sus componentes, relaciones y procesos" (AponteGarcía, Escobar-Ocampo, Molina-Saldarriaga, 2018: 46). En consecuencia, esta instancia del CPU resulta clave para integrar y realizar una interpretación valorativa de la información recolectada en las fases anteriores desde la posibilidad de producir aperturas a la ciudadanía y arribar al establecimiento de juicios de valor, según "grupos de población que difieren en sus preferencias estéticas" (Muñoz-Pedreros, 2004: 147), funcionales, significativas y simbólicas.

En el Parque Sarmiento, la participación activa y comprometida de sus habitantes - permanentes o temporarios- constituye una contribución sustancial, la cual es recabada mediante el taller de valoración y participación ciudadana: Paisaje como lenguaje sensible (Riveros y Períes, 2018). Al mismo tiempo, es de vital importancia conocer las impresiones y opiniones de especialistas en paisaje y de un grupo de profesionales multidisciplinares, ya que portan información calificada con perspectiva disciplinar y multidisciplinar. Metodológicamente, se opera por medio de distintos modelos y técnicas de consulta -presenciales o virtuales-, elaborados de modo particular para cada grupo. 
Las consultas de valoración son realizadas en correspondencia con las categorías y subcategorías del sistema de categorización de componentes paisajísticos, por su desagregación (Delgado y Pantoja, 2016) —en algunos casos se hace referencia específica a componentes particulares de las subcategorías, por su singularidad excepcional-. En la consulta a profesionales multidisciplinares y especialistas en paisaje, además, se hace mención especial al valor que predomina en cada área homogénea, correspondiente con los siguientes tipos: a) estético, por predominio de componentes paisajísticos (CP) que por sus características transmiten belleza y armonía; b) ecológico, por predominio de $\mathrm{CP}$ que contribuyen a la biodiversidad y equilibrio ambiental; c) productivo, por predominio de $\mathrm{CP}$ con capacidad para proporcionar beneficios económicos, por medio de actividades culturales, comerciales, turísticas, industriales; d) histórico, por predominio de CP que se presentan como testimonios o huellas del pasado; e) social, por predominio de $\mathrm{CP}$ que propician el uso y la apropiación de los habitantes; f) identitario, por predominio de CP que despiertan sentimientos de pertenencia en un determinado grupo social.

La información cuantitativa que resulta de la síntesis de los datos recabados de los tres modelos de consulta se concreta como producto de esta fase en una serie de múltiples gráficos estadísticos, correspondientes con cada área homogénea y con cada tipo de información recabada, como se ejemplifica en las figuras 7 y 8 . Estos gráficos permiten conocer sobre el agrado o desagrado respecto del paisaje - en aspectos globales-, el valor positivo y negativo de sus componentes, el tipo de valor que caracteriza al paisaje de cada área $\mathrm{y}$, por parte exclusiva de los especialistas en paisaje, su calidad en cada área y las acciones de fortalecimiento que demanda cada valor.

Figura 7. Síntesis de la valoración de especialistas en paisaje sobre la calidad y los componentes del paisaje en el AH1

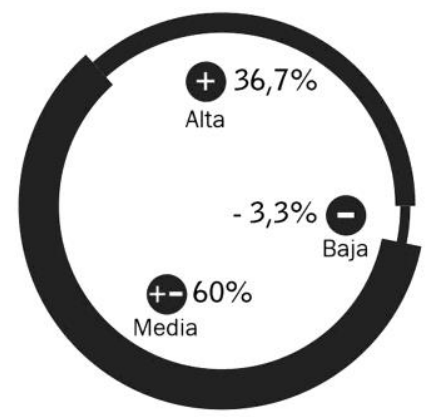

Valoración de calidad del paisaje

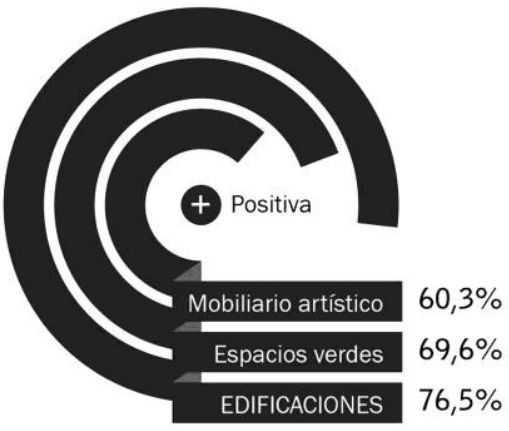

Valoración de los componentes del paisaje

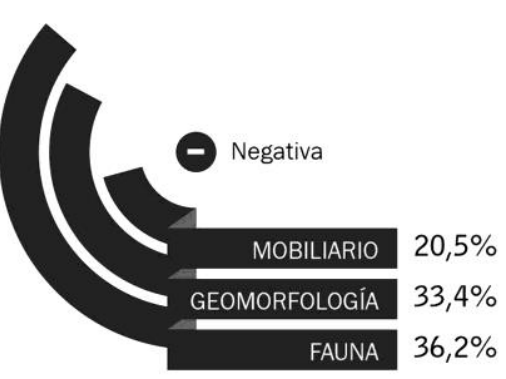

Fuente: elaboración propia. 
Figura 8. Síntesis de la valoración ciudadana de los componentes paisajísticos del AH1

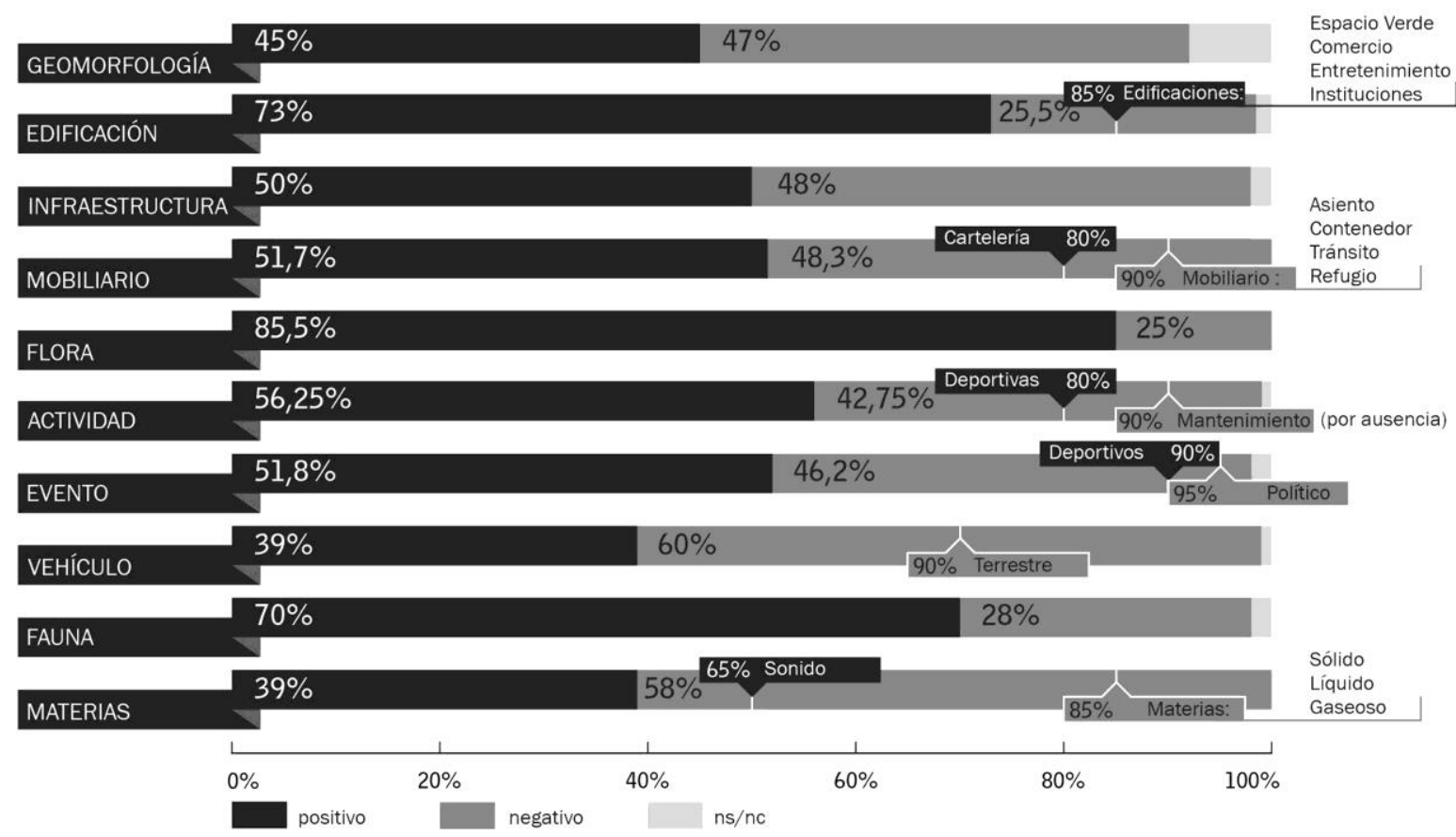

Fuente: elaboración propia.

\section{La fase de evaluación}

La evaluación es una consecuencia del proceso desarrollado durante la construcción del catálogo del Parque Sarmiento. En función de las coincidencias que emergen de las diferentes consultas, con altos porcentajes de valores positivos o negativos, se confronta con la caracterización de las áreas, la identificación de componentes paisajísticos y la interpretación territorial, a fin de precisar aspectos o atributos y los correspondientes indicadores que explican dicha valoración para establecer el tipo de valor del paisaje de cada área -desde la interpretación objetiva del equipo de investigación-.

El valor es enunciado, argumentado y fundamentado de modo escrito, y se representa visualmente con la técnica gráfica de collage. De este modo, se componen imágenes representativas de los elementos que caracterizan y determinan el valor del área, por su significado, protagonismo o predominancia. Las figuras 9 y 10 lo ejemplifican. 
Figura 9. Collage representativo del paisaje del AH1

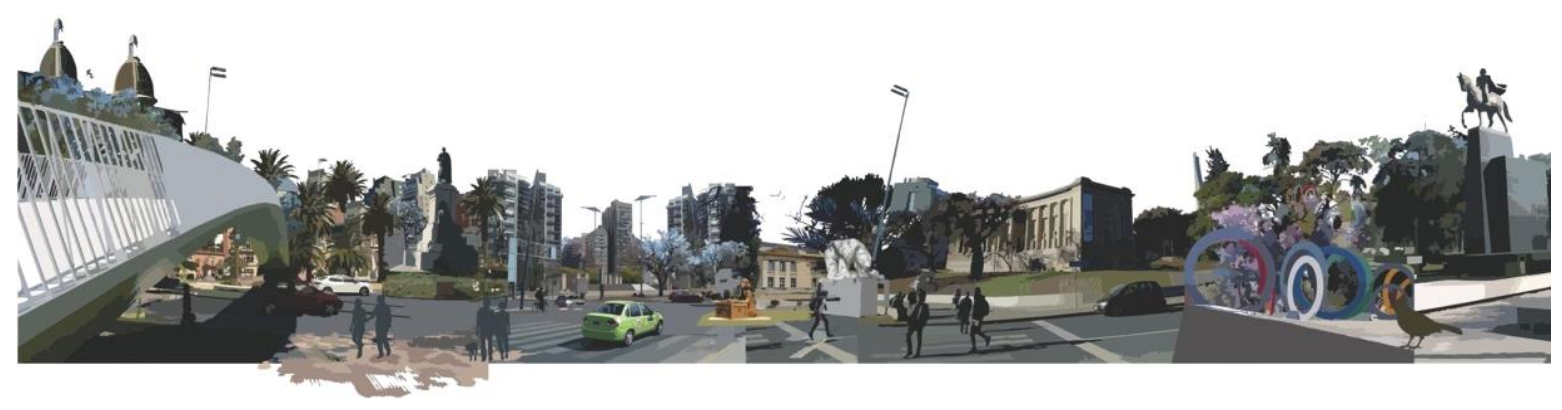

Fuente: elaboración propia.

Figura 10. Collage representativo del paisaje del AH2

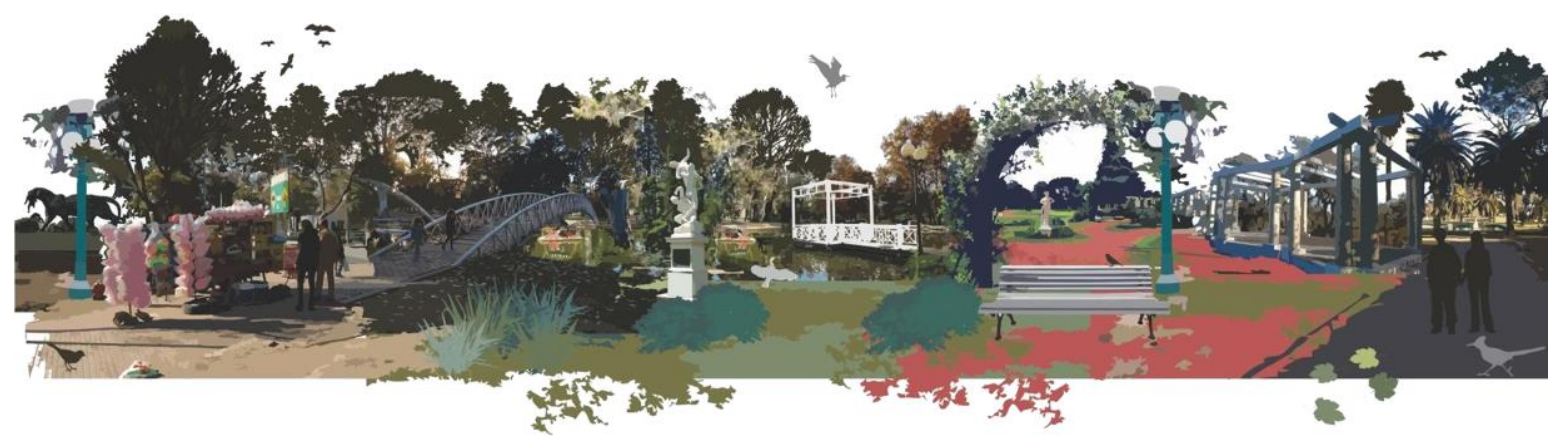

Fuente: elaboración propia.

Los tipos de valores establecidos para las cinco áreas del Parque Sarmiento se sintetizan en las siguientes expresiones:

- El paisaje del área homogénea (AH) 1 se define por su valor histórico y está compuesto por los siguientes elementos representativos: las edificaciones que constituyen el componente principal, integrado por la subcategoría cultura, con los tipos museo, centro cultural, biblioteca y la subcategoría espacios verdes, con los tipos plaza, plazoleta, paseo y parque. Además, se evidencia el mobiliario artístico con los tipos escultura, mural, estatua, fuente y mástil.

- El paisaje del AH2 se define por su valor identitario y está compuesto por los siguientes elementos representativos: los espacios verdes que constituyen el componente principal, conformado por los tipos: jardines, paseos, plaza, plazoleta. Le siguen la flora y el recurso hídrico, con el tipo laguna (Laguna del Jardín Thays); además se presenta mobiliario artístico con los tipos escultura, mural, estatua y fuente; y la fauna. 
- El paisaje del AH3 se define por su valor social y está conformado por los siguientes elementos representativos: los espacios verdes que constituyen el componente principal, formado por los tipos: jardín, plazoleta, paseo y boulevard. Le siguen la flora y la geomorfología, con el tipo barranca, así como las actividades deportivas y recreativas y los eventos culturales y deportivos. Se destaca a la noria como el mobiliario artístico representativo y singular incluido en esta área.

- El paisaje del AH4 se define por su valor histórico y está compuesto por los siguientes elementos representativos: el Teatro Griego constituye el componente principal, junto a los espacios verdes, con los tipos jardín, paseo, plaza, plazoleta y el mobiliario artístico. Le siguen las actividades de tipo deportiva y recreativa y los eventos de tipo deportivo y cultural. Se destaca la geomorfología, con el tipo barranca (Barrancas del Paseo Templete del Amor).

- El paisaje del AH5 se define por su valor productivo y está compuesto por los siguientes elementos representativos: las edificaciones constituyen el componente principal, integrado por las subcategorías educación, administración, transporte, residencia colectiva, salud. Le siguen la flora y la infraestructura.

A partir de la fundamentación del valor, se establecen las "consideraciones de calidad paisajística" correspondientes a cada área y las respectivas acciones que demandan para la sostenibilidad del paisaje. Los tipos de acciones establecidos son: a) preservar lo que se requiere proteger, conservar o regular; b) recuperar lo que se necesite reparar, remediar, sanear, restaurar o reconstruir; c) potenciar aquello que es necesario acondicionar o refuncionalizar; d) incorporar lo que es necesario incrementar, implementar o introducir; e) suprimir lo que sea preciso interrumpir, restringir, prohibir, remover, erradicar, desalojar o eliminar.

Estas acciones se plantean para orientar el desarrollo de políticas, planes y proyectos particulares - los que debieran ser formulados, diseñados y ejecutados por profesionales especialistas en cada campo de conocimiento y de modo interdisciplinar- para fortalecer el carácter que manifiesta cada área homogénea. Las consideraciones de calidad paisajística, con sus consecuentes acciones, argumentan aspectos y medidas generales, las que deben vincularse y detallarse con la información expuesta en el desarrollo de las fases de identificación y caracterización. 


\section{El CPU como producto}

La conjunción de los contenidos desarrollados en cada fase del CPU, y en correspondencia con sus tres años de construcción, da por resultado un documento de información múltiple. Se trata de un producto que permite conocer los recursos naturales y culturales con los que cuenta un sector de ciudad, así como comprender su dimensión histórica, reconocerlo, interpretarlo, caracterizarlo y valorarlo, para, finalmente, establecer líneas de acción tendientes a su protección y proyección sostenible —en términos de calidad y cualidad paisajística-.

El estudio se concreta en un objeto editorial, bajo el formato de libro (Períes, Kesman, Barraud, 2019b). Esta publicación se orienta a quienes cuentan con poder de decisión y acción y, al mismo tiempo, a un público general con la intención de permear en la sociedad los valores del espacio público tratado para su salvaguarda colectiva. Respecto al producto obtenido, su originalidad se relaciona con la ausencia de antecedentes encontrados en cuanto a la aplicación de catálogos del paisaje en territorios urbanos y, particularmente, como estudio prospectivo de espacios públicos.

\section{Discusión}

Sobre los antecedentes con los que se pueden establecer relaciones comparativas, se señalan dos tipos. Por un lado, aquellos que coinciden metodológicamente con las estrategias empleadas, a pesar de que se diferencian en las escalas de abordaje y se abocan a la planificación a escala territorial, como "Los catálogos del paisaje de Cataluña", desarrollados con intención de contribuir a la protección, la gestión y la ordenación del paisaje a escala de las comarcas de Cataluña. "Esos catálogos se conciben normativamente como herramientas útiles para ordenar y gestionar el paisaje desde la perspectiva del planeamiento territorial" (Nogué, Sala y Grau, 2016: 16).

La coincidencia reside de manera puntual en lo que al ordenamiento y a la gestión respecta; los mencionados catálogos se presentan como "documentos de carácter descriptivo y prospectivo que determinan la tipología de los paisajes de Cataluña, identifican sus valores y su estado de conservación y proponen los objetivos de calidad que deben cumplir" (Nogué, Sala y Grau, 2016: 15). La diferencia más sustancial y sobre la escala de abordajes se encuentra en la ausencia de legislación para nuestro contexto, que para el caso de la Comunidad Europea le otorga al catálogo carácter vinculante y compromiso de implementación y cumplimiento. 
Respecto a la comparación de fases de la investigación, en la instancia de interpretación, que comprende desde la globalidad de la ciudad hacia la zona de estudio, pueden establecerse parangones con el trabajo desarrollado por Solís y Troitiño que pertenece al campo de la geografía y aborda "la relación entre economía, política y territorio" (Solís y Troitiño, 2012: 144) desde una interpretación con foco en los procesos escalares de producción, urbanización y regulación. El andamiaje que sostiene la producción es el paradigma de la red relacional que "propone un marco de interpretación y una plataforma descriptiva y de conocimiento orientada a la acción. La idea de red se concibe como herramienta" (Solís y Troitiño, 2012: 158) y aporta la noción de “reescalamiento" (Solís y Troitiño, 2012: 150).

En ambos casos - a pesar de que las escalas involucradas en los respectivos estudios son diferentes, pues en el Parque Sarmiento la escala de interpretación es la ciudad, y en el caso europeo es la región-, se definen acercamientos escalares como aproximación al entendimiento integral de la zona de estudio y en consecuencia con el enfoque paisajístico que involucra a la globalidad y a las partes de modo simultáneo.

Por otra parte, la investigación puede discutirse con trabajos que abordan aspectos del mismo caso de aplicación; por ejemplo, con las cuestiones expuestas en la investigación de Ferreyra (2015), pues hay coincidencia con su abordaje respecto a la necesidad de la recuperación del parque dependiente de un proyecto integral que acuda a sus valores originales. El autor refiere a ello del siguiente modo:

\footnotetext{
La recuperación del Parque Sarmiento sólo es posible a partir del desarrollo de un proyecto integral que contemplando los valores vigentes de su patrimonio cultural y natural, establezca los lineamientos para el diseño de una política pública orientada a redefinir su rol e imagen dentro del sistema de espacios abiertos públicos de la ciudad de Córdoba (Ferreyra, 2015: 144).
}

A pesar de esos puntos de contacto, la principal diferencia reside en que el trabajo de Ferreyra propone un proyecto de recuperación sustentado en la utilización de la clásica estructura de análisis DAFO (debilidades, amenazas, fortalezas y oportunidades), mientras la investigación desarrollada con el CPU define una estrategia procedimental de enfoque cualitativo y estadístico -en correspondencia con las fases que estructuran el propio método, con sus técnicas y procedimientos aquí expuestos-, con la que se contribuye al conocimiento del estado y valor paisajístico del espacio público desde herramientas que aportan rigor científico, para contribuir al ordenamiento y desarrollo del parque, considerando el valor patrimonial desde el enfoque paisajístico y con una adecuación a las demandas sociales actuales. 


\section{Conclusiones}

Esta investigación deja como huella dos aportes fundamentales, ambos inherentes al conocimiento del paisaje: la construcción del catálogo del Parque Sarmiento y la consolidación de una metodología para la ejecución de los CPU. En lo que respecta a la metodología, el trabajo desarrollado ha requerido una sustancial planificación, así como también ha demandado el diseño específico y el ensayo reiterado de múltiples protocolos y herramientas (tanto conceptuales como operativas, digitales y analógicas), que en muchos casos son inéditas.

Esta redefinición del método como evolución, se refiere a su concepción y crecimiento durante las investigaciones aplicadas por el equipo en ediciones anteriores ${ }^{7}$ -con otra escala de espacio urbano y estructura de corredor lineal-. Cada una de las fases que integran la construcción del catálogo fue readecuada a las nuevas condiciones de la zona de estudio, tanto desde la teoría como desde la praxis científica. Como derivación de esta labor, el producto resulta superador de las experiencias predecesoras.

En relación con el Parque Sarmiento, la posibilidad de conocer a profundidad sus componentes paisajísticos, que en su interrelación le otorgan su peculiar carácter y valor paisajístico, se considera una oportunidad para la redefinición de su rol recreativoeducativo y cultural, y principalmente de su valor patrimonial integral -que lo ha convertido en Monumento Histórico Nacional-, junto al medioambiental. Por ello, este estudio, devenido en informe técnico, es transferido a los gobiernos locales para su potencial implementación en el diseño de políticas públicas, programas y proyectos de diseño.

De esta manera, el catálogo de paisaje del Parque Sarmiento contribuye a la planificación urbana -con una mirada amplia del problema que trasciende, incluso, al propio parque - si se entiende que su implementación involucra la diversidad de acciones y la participación de personas que desde sus roles representan a entes públicos y privados, y primordialmente apunta a la conjunción de lineamientos estratégicos direccionados al futuro de la ciudad.

\footnotetext{
${ }^{7}$ Trabajos publicados en los libros Catálogo del paisaje del río Suquía en la ciudad de Córdoba, Vol. 1: centro y pericentro noroeste y Vol. 2: suburbano noroeste (EDUCC, 2012 y 2016).
} 


\section{Referencias}

Aponte-García, G.; Escobar-Ocampo, L. M.; Molina-Saldarriaga, C. A. (2018). Exploración de metodologías para la valoración del paisaje. Aproximación al diseño de una metodología propia. Bitácora Urbano Territorial 28 (1), pp.45-60. DOI: https:// doi.org/10.15446/bitacora.v28n1.56700

Busquets, J.; Cortina, A. (Ed.) (2009). Gestión del paisaje: manual de protección, gestión y ordenación del paisaje. Barcelona: Ariel.

Busquets, J (Coord.) (2007). Per una corretta gestione del paesaggio: linee guida. Barcelona: Direcció General d'Arquitectura i Paisatge, Generalitat de Catalunya.

Consejo de Europa (2000). Convenio europeo del paisaje. Florencia: Consejo de Europa.

Fernández, R.; Plaza, J. I. (2019). Participación ciudadana y educación en materia de paisaje. Cuadernos Geográficos 58 (2), pp. 262-286. DOI: http://dx.doi.org/10.30827/cuadgeo.v58i2.7429

Ferreyra, M. (2015). Estrategia de recuperación del parque Sarmiento, ciudad de Córdoba, Revista Pensum, 1, pp. 144155.

Muñoz-Pedreros, A. (2004). La evaluación del paisaje: una herramienta de gestión ambiental. Revista chilena de historia natural, 77(1), 139-156. DOI: https:/ / dx.doi.org/10.4067/S0716-078X2004000100011

Nogué, J.; Sala, P.; Grau, J. (2016). The landscape catalogues of Catalonia. Barcelona: ATLL.

Nogué, J.; Sala, P.; Grau, J. (2016). Los catálogos de paisaje de Cataluña: Metodología. Barcelona: ATLL.

Page, C. (1996). El Parque Sarmiento. Colección Historia de la Arquitectura de Córdoba. Fundación Centro: Río Ceballos.

Períes, L.; Ojeda, B.; Kesman, C. (2016). La identificación de componentes paisajísticos para catálogos del paisaje urbano. Projetar, 8, pp. 73-89.

Períes, L.; Kesman, C.; Barraud, S. (2019a). El color como componente paisajístico en los catálogos de paisaje urbano. Revista de Arquitectura, 22 (1). DOI: https:// doi.org/10.14718/RevArq.2020.2824

Períes, L.; Kesman, C.; Barraud, S. (2019b). Catálogo de paisaje del Parque Sarmiento de la ciudad de Córdoba. Córdoba: EDUCC.

Delgado, A. M. y Pantoja, F. (2016). Valoración del paisaje en una propuesta de turismo sostenible: la Ruta del Oro. Nariño (Colombia). Cuadernos de Geografía: Revista Colombiana de Geografía 25 (1): 233-253. DOI: http://dx.doi.org/10.15446/rcdg.v25n1.50157

Porcal-Gonzalo, M. C. (2019). Búsqueda de especificidades en el carácter de los paisajes del viñedo e identificación de sus procesos de patrimonialización. Cuadernos Geográficos, 58(2), 215-239.

Riveros, R. y Períes, L. (2018). Valoración sensible del paisaje urbano: La experiencia del Parque Sarmiento en la ciudad de Córdoba. Arquetipo, 16, pp. 97-111.

Solís Trapero, E. y Troitiño Vinuesa, M. A. (2012). El paradigma de la red: Bases para una nueva interpretación del territorio y de los procesos escalares de la urbanización. Boletín de la Asociación de Geógrafos Españoles, 60, pp. 141-164. DOI: https://doi.org/10.21138/bage.1502

Swanwick, C. (2002). Landscape character assessment: guidance for England and Scotland. Edinburgh: The Countryside Agency \& Scottish Natural Heritage. 


\section{Agradecimientos}

Se agradece de manera especial a María Cecilia Kesman quien participó del desarrollo de la investigación como Codirectora. Se agradece al equipo de profesionales que ha colaborado en distintas instancias del desarrollo de la investigación: Art. Plástica Martha Bersano; Dis. Industrial Mauro Bianchi; Arq. Natalia Brizuela; Mgter. Geóloga Cristina Chávez; Mgter. Bióloga Cecilia Eynard; Mgter. Ing. Agrónoma Diana Perazzolo; Mgter. Arq. Julio Rebaque de Caboteau y Biólogo Diego Alejandro Serra. También a la entidad financiadora y evaluadora del proyecto de investigación, por la adjudicación de fondos para la ejecución del trabajo. 